\title{
SOBRE AS ESPÉCIES DE Eucinostomus BAIRD \& GIRARD IN BAIRD, 1855, QUE OCORREM EM ÁGUAS BRASILEIRAS (ACTINOPTERYGII, PERCIFORMES, GERREIDAE)
}

\author{
About Eucinostomus Baird \& Girard in Baird, 1855 \\ that they occur in brazilian waters (Actinopterygii, \\ Perciformes, Gerreidae)
}

José Vanderli Andreata ${ }^{1}$

\section{Resumo}

O trabalho tem como objetivo analisar as espécies do gênero Eucinostomus Baird \& Girard in Baird, 1855, que ocorrem em águas brasileiras, com base no exame de material tipo, nos protólogos e coleções procedentes de diversas localidades do Brasil. Foram examinados a morfologia externa, o esqueleto cefálico e os otólitos sagitta. A comparação entre o material tipo e os espécimes de diferentes coleções mostrou a semelhança entre algumas espécies do gênero que são sinonimizadas. É proposta a revalidação de Eucinostomus aprion (Cuvier, 1829) e uma nova combinação denominada Eucinostomus lefroyi (Goode, 1874). A distribuição geográfica e alguns aspectos ecológicos também são abordados.

Palavras-chave: Gerreidae; Eucinostomus; Brasil.

\section{Abstract}

The aim of this research was to evaluate species of the genus Eucinostomus Baird \& Girard in Baird, 1855 occurring in Brazilian waters, based on the examination of type-material and specimens collected throughout the country. The external morphology, the cephalic skeleton and sagitta otoliths were examined. A comparison between type-material and that collected from several sites has shown that some species were synonymized. It is proposed that Eucinostomus aprion (Cuvier, 1829) be re-evaluated and a new combination Eucinostomus lefroyi (Goode, 1874) be denominated. The geographic distribution and some ecological aspects were also approached.

Keywords: Gerreidae; Eucinostomus; Brazil.

Ph.D., Professor no Instituto de Ciências Biológicas e Ambientais, Universidade Santa Úrsula, Rua Fernando Ferrari, 75, Botafogo, Rio de Janeiro - 22231-040; jvandreata@gmail.com 


\section{Introdução}

A família Gerreidae, da ordem Perciformes, compreende cerca de 40 espécies e quinze gêneros $(1,2,3,4,5,6,7)$. O gênero Eucinostomus é caracterizado pelo corpo baixo, ossos operculares lisos, escamas ciclóides na cabeça e ctenóides e ciclóides no corpo. Apresentam ampla distribuição geográfica, ocorrendo no Atlântico Norte e Sul, desde New Jersey até Montevidéu, inclusive no Mar das Antilhas, Golfo do México, Bermudas e Oeste da África. No Pacífico, ocorre no México, Costa Rica e de El Salvador ao Peru.

Eucinostomus foi tratado por Andreata $(8,9,10)$ como um sinônimo de Gerres por não considerar a presença do segundo espinho anal mais grosso do que o terceiro e a altura do corpo como caracteres relevantes para mantê-los como gêneros independentes.

Neste trabalho, são avaliadas as espécies brasileiras de Eucinostomus, com base em um grande número de coleções provenientes de regiões geográficas distintas enfocando-se a morfologia externa, a distribuição geográfica, o esqueleto cefálico e o otólito sagitta. Considera-se para o Brasil a ocorrência das espécies Eucinostomus aprion, E. Melanopterus, E. gula e E. lefroyi.

\section{Material e métodos}

O material estudado é proveniente de coletas realizadas em diversas regiões brasileiras e de coleções nacionais e estrangeiras. Os métodos utilizados na captura dos espécimes foram redede-arrasto de porta, rede-de-espera, arrasto-de-praia (picaré) e puçá. Todo material coletado foi registrado na coleção da Universidade Santa Úrsula sob a sigla USU. Cada lote é precedido da sigla da Instituição, seguido do número de espécimes, entre parênteses.

O esqueleto cefálico foi corado com alizarina S vermelha segundo Taylor (11) e para os otólitos segundo Nolf (12) e Chao (13). O desenho do esqueleto cefálico foi feito em câmara clara apenas para Eucinostomus aprion, em virtude da grande semelhança entre as espécies estudadas, e os otólitos para todas as espécies tratadas. A salinidade foi aferida com refratômetro e o conteúdo estomacal examinado de exemplares coletados segundo a metodologia de Zavala-Camin (14).

\section{Resultados}

Eucinostomus aprion (Cuvier, 1829) combinação nova.

Gerres aprion Cuvier, 1820 (Localidade típica: Havana, Martinica e Montevidéu).

Eucinostomus argenteus Baird \& Girard in Baird, 1855: 345 (Localidade típica: Beeley's Point, New Jersey, USA).

Diapterus gracilis Gill, 1862: 246 (Localidade típica: Cape San Lucas).

Eucinostomus gula Goode, 1876: 39 (Localidade típica: Brasil).

Eucinostomus gula 1876 (P.P.)

(Localidade típica: Martinica)

Gerres jonesii Günther, 1879: 389

(Localidade típica: Bermudas).

Eucinostomus harengulus Goode \& Bean, 1879: 132; Andreata, 1979: 248, (Localidade típica: West Florida).

Gerres gracilis Jordan \& Gilbert, 1882:

329 (Localidade típica: Cape São Lucas).

\section{Descrição e comentários}

Corpo baixo e comprimido ligeiramente no dorso, a altura está contida 2,8 a 3,3 vezes no comprimento padrão. Cabeça contida 3,1 a 3,5 vezes no comprimento padrão. Focinho maior que nas demais espécies do gênero. Perfil anterior uniforme e quase reto. Sulco pré-maxilar contínuo ou interrompido parcialmente por uma faixa de escamas; 7 - 8 rastros na parte inferior do primeiro arco branquial; 38 - 49 escamas na linha lateral. Nadadeira anal III $+7-8$. Nadadeira dorsal com pigmentação escura nos espinhos. Nadadeira caudal com 6 - 11 raios acessórios epaxiais e 6 - 10 hipaxiais; três cecos pilóricos. Denominado popularmente de carapicú e carapicú-açu (Figura 1). 


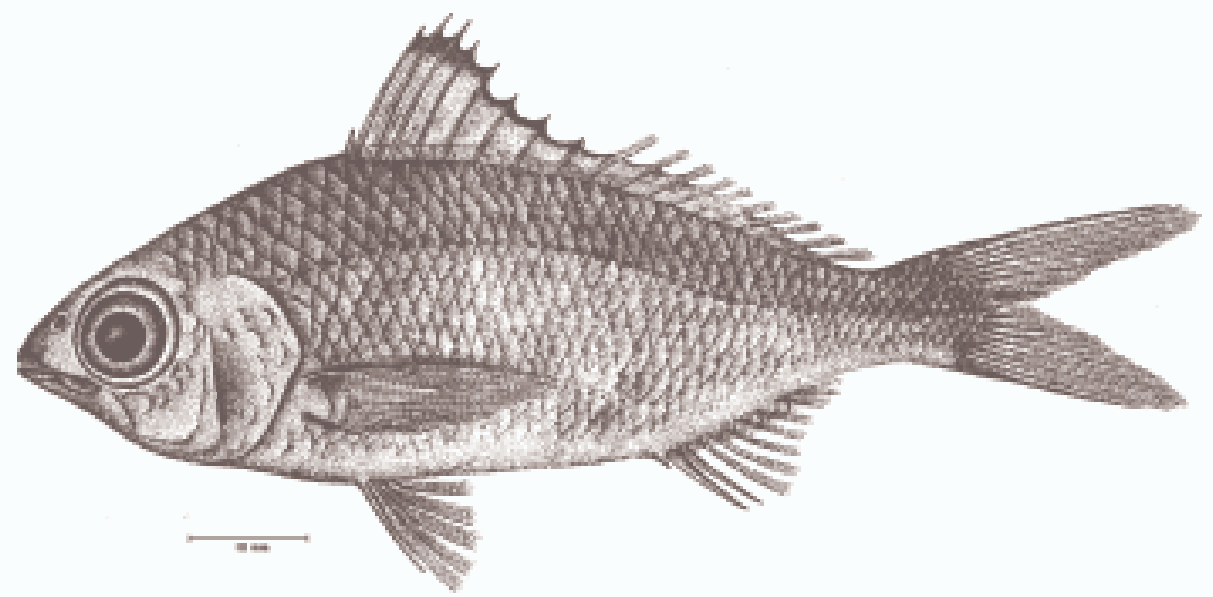

\section{FIGURA 1 - Vista lateral de Eucinostomus aprion \\ Fonte: Cuvier, 1829.}

Apresenta ampla distribuição geográfica, ocorrendo no Atlântico norte e sul, desde New Jersey até Montevidéu, inclusive no Mar das Antilhas, Golfo do México e Bermudas. No Pacífico de El Salvador ao Peru.

Verificou-se nas coleções examinadas que a altura do corpo varia no material estudado medindo os maiores exemplares 280 $\mathrm{mm}$ de comprimento total. Segundo Menezes
\& Figueiredo (15), podem atingir $300 \mathrm{~mm}$. Nos exemplares de Galápagos (Pacífico), notouse que o diâmetro dos olhos e o corpo mais baixo do que naqueles do Atlântico. É a espécie mais comum encontrada no litoral brasileiro.

O esqueleto cefálico (Figura 2) apresenta grande semelhança e a mesma estrutura das demais espécies de Eucinostomus (10).

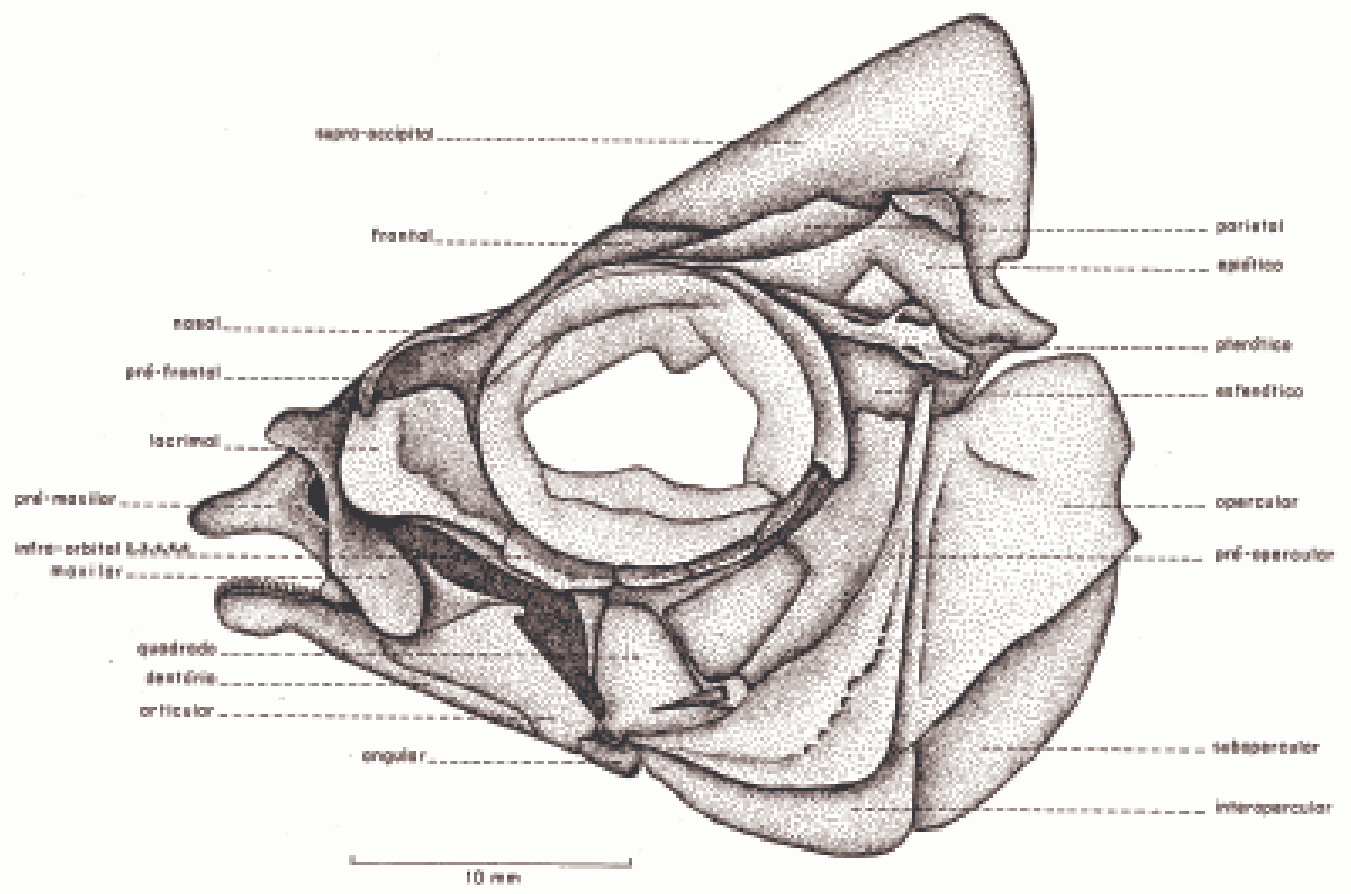

FIGURA 2 - Vista lateral do esqueleto cefálico de Eucinostomus aprion

Fonte: Cuvier, 1829. 
O otólito sagitta é opaco e robusto, de forma elíptica, comprimento maior do que a largura, margem ventral recortada, ostium raso se tornando mais aprofundado próximo à região ventral, sulco longo e profundo $(9,10)$ (Figura $3 \mathrm{a}, \mathrm{b})$.

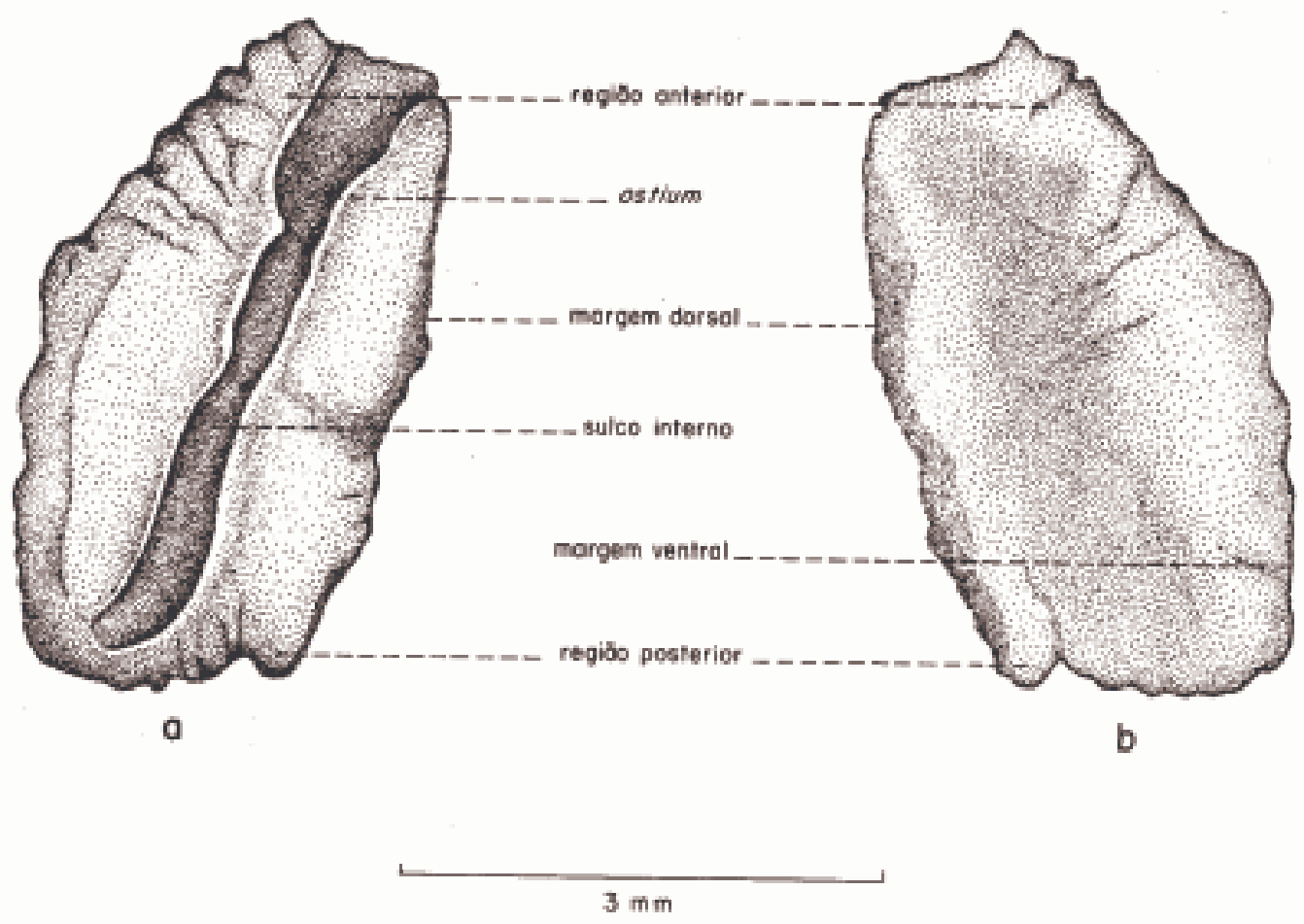

\section{FIGURA 3 - Vista lateral interna (a); vista lateral externa (b) do otólito sagitta de Eucinostomus aprion}

A análise do conteúdo estomacal revelou a presença de anelídeos (poliquetas), pequenos moluscos, crustáceos (anfípodes), algas, vasa orgânica. Quanto à salinidade, é a espécie que mais tolera uma grande variação, entre 15,56 a 36. Segundo Springer e Woodburn (16) foi encontrada em salinidade de 1 .

Jovens e adultos apresentam manchas e barras verticais na parte superior do corpo, no entanto, quando o animal está sob estresse em aquário, estes desaparecem e retornam imediatamente; quando fixados em formol são ausentes

\section{Material examinado}

Bermudas - Síntipos de Gerres jonesii, BMNH 1879 1.8.9-1.14 (2); 10/VIII/1948, FMNH 48324 (3); Nonsuch Islandl Cashe Hasbor, Guar- net Rock seeps Myzs, Berm udas Oceanogr. Expedsl 1829-1932 AMNH 52441 (6). Estados Unidos Síntipos de Eucinostomus harengues USNM 5145 (2), west Florida: lectotipo USNM 8159 (1), paralectótipos USNM 201674 (12) e paralectótipo USNM 32491 (1) de Eucinostomus argenteus Beeleyls Pointl New Jersey, USA; Floridal Matanzas River Marylanel 01/VII/19671 AMNH 56046 (18); Co. Key Biscaynel E. end Bear cut Bridgel Miamil 15/Xl/19641 AMNH 20605(2); Miamil near Seaquariuml 26/XI/19571 AMNH 56047 (9); Sudlow Creekl Floridal 26/XII/19481 FMNH 78525 (10); Barrow pit air strip cedarkey, 14/IXI19571 MZUSP não cat.(3); $34^{\circ} 381 \mathrm{~N} 176^{\circ} 591 \mathrm{~W}$ to $34^{\circ} 381 \mathrm{~N} 1$ $77^{\circ} 011 \mathrm{~W} 1$ 17/IXl19821 AMNH Uncat (2); $34^{\circ} 12^{\prime}$ IN1 $77^{\circ} 431 \mathrm{~W}$ to $34^{\circ} 11 \mathrm{IN}, 77^{\circ} 41 \mathrm{IW}, 18 / \mathrm{IXI} 1982$, AMNH Uncat (1); North Carolinal 24/Xl19641 MCZ 34180 (6); Georgial $31^{\circ} 51 \mathrm{~N} 181^{\circ} 0812^{\prime} \mathrm{W}$ Uncatl AMNH (1); South Carolina Geogetow countryl North Inletl Crabhaul Creek, $31^{\circ} 211$ N1 $79^{\circ} 11 \mathrm{IW}, 28 / \mathrm{IX119681}$ 
AMNH 73786 (3); Virginia, Rudee Inletl Virginia Beachl 23/VI/19761 AMNH Uncat (18) Virginia Key, 30/IXl1964, AMNH 56048 (15); Carteret Co. Bogue Sound Lagoon, Atlantic Beachl North Carolina, 13/ VIII/19691 AMNH 65203 (1); Sarasota Co.1 N. Lemon Bayl Floridal 02/VII/1966, AMNH 56050 (56); Dry Tortugas, 1/19031 AMNH 56052 (1); Butternut Keyl Coast West, Floridal AMNH 56053 (1); West Coast, Florida, 03/I/19411 AMNH 56054 (1); New Jersey, Brigantine, Abrecon Inll cove at end of Islands, 19/1X/1973, AMNH 56058 (19).

Golfo do México - Lat. 29²8'3" N, Long. $88^{\circ} 14$ 'W, Oregon 10/IV/1980, TCWC 2932.1 (9). México Sonora, Punta Penasco, 20/VII/1946, AMNH 56049 (5). Cuba - Paralectótipo de Gerres aprion MNHN 9456 (1); Havana Marke 22/II/1912, AMNH 3380 (1). Costa Rica - Porto Culebra, Eastern Pacific, 24/I/1938, AMNH 56064 (1); Gulf of Dulce, Golfito, Eastern p cific, AMNH 56059 (6). Haiti - Port/Prince, 1949 - 1950, AMNH 37123(5). EI Salvador -Gulf of Fonseca, Meanguara Island, AMNH 56066 (3); Ilhas Virgens - Virgin Gorda sound $18^{\circ} 29^{\prime} \mathrm{N}, 64^{\circ} 24^{\prime} \mathrm{W}$, 13-14/194 AMNH 1743(1); NW of Prettyklip Point, St John Bay, appro $18^{\circ} 20^{\prime} 13^{\prime \prime} \mathrm{N}, 64^{\circ} 50^{\prime} 57^{\prime \prime} \mathrm{W}$, usvi St. Thomas, 12/XI/1966, GCI 2393(3); Ecological Research Station, VIII/1967, AMNH 56045 (1). Porto Rico - Paio Seco, VII/1914, AMNH 8910 (67). Panamá - Caledonia, San Bras, 06/V/1924, AMNH 11419 (18); Balbo, Patilla airstrip., Pacific, 10/I/1978, AMNH 74048 (43); Limon Bay ne, entrance to Fort sherman, approx. 9 $21^{\prime} 32^{\prime \prime} \mathrm{N}, 7^{\circ} 56^{\prime} 53^{\prime \prime} \mathrm{W}$, Atlant Coast Panama, 13/XII/1966, GCRL 2399(31); 23/ III/1963, USN, 247256 (2); Galeta Is (Atlantic), MCZ 43506 (1); Jungle Point, MCZ 43493 (1); Taipc Club Board Basin, Atlantic coast (fresh watter), 24/VI/ 1968, MC 46674 (4); Isla Pedro Gonzales, Pacific, 16/I/1978, AMNH 74045 (1); Islas Perlas, Islands senor, 29/VII/1933, AMNH 56051 (173) Bahia Honda, Eastern Pacific, AMNH 56060 (19). Dominica - Dept. St. george, Inshore shelf 3,5 km Rogean Cente 16/VII/1977, AMNH 36651 (2); Portsmouth, 25/VI/1912, AMN 17042 (1), AMNH 17042 (1); Dept. St. George, shelfoffcharlotte villt 18/VII/1977, AMNH 36702 (3). Martinica Lectótipo de Gerres aprion, MNHN 5665 (1); Paralectótipo de Gerres aprion MNHN 9455 (1) Curaçao - Ductch W. I., 08/X/1909, AMNH 291 (1). Barbados - MCZ 59760 (1). Venezuela - N. Esparta, Isla Margarita, Boca dei Rio, 20/II/1977, GCF 15801 (5); Isla Cubagua, Pta. Charagato to Pta. Brasil, Nueva Espart 16/II/1977, GCRL 15679
(2); Isla Larga, punta Este dei Archipélago Los Roques, Dependencio Federal, 05/VIII/1963, USU 00410 (4). Colômbia - Isla de San Bernordo, ICN 296 (4). Equador - Santo Rosa, 05/1X/1962, NMC68-1944 (1); San Salvador, Galápagos Islands, Sullivn Braz, 22/I/1978, AMNH 74044 (1). Peru Estero de Tumbes, isla del Culebro, Tumber, 23/ II/1974, GCRL 12654 (12); Calloa, MCZ 45209 (4). Brasil - Síntipo de Eucinostomus gula MNHN 9462 (2) (in part); MNHN B 2681 (1); MNHN não cat. (3); MNHN 489(3); 16/III/1874, ZMK 476 (1). Maranhão - $01^{\circ} 32^{\prime} 5 ; 43^{\circ} 47^{\prime} \mathrm{W}$ em frente à Ilha Cotindibo, 07/IV/1957, MZUSP não cat. (1). CearáMorro Branca, próximo de Beberibé, 1972, MZUSP 720779(2); Rio Coco, Fortaleza, III/1945, MNRJ 10868 (5). Alagoas - Mercado de Maceió, MZUSP não cat. (1). Sergipe - Aracaju, VII/1961, MZUSP não cat. (1); Rio Sergipe em Chico Chaves, próximo a Aracaju, MZUSP não cat. (5). Bahia - Mus. Geneve, MNHN 985 não cat. (1); MNHN não cat. (1); MNHN 2805 não cat. (2); MNHN 151 não cat. (1); Playas Arembepe, obout $1 \mathrm{~km}$ sot town $12^{\circ} 45^{\prime} 00^{\prime \prime} \mathrm{S}$, 38¹0'00"W, Salvador, 30/IV/1973, GCRL 10870 não cat. (2); idem, 26/VIII/1972, GCRL 9405; Arembepe, 26/VIII/1972, MZUSP não cat.(1); Perto da Tibros off send of Parque Interlagos, Salvador, 01/V/1973, GCRL 10818 (12); Parque Interlagos 91/V/1973, MZUSP 10297 (24); Pontal, Ilhéus 25/X/1971, MZUSP 10296 (9); Barra do Paraguassu de Maragogipe, 10/XII/1974, MZUSP não cat. (3); Mercado São Salvador, 16/IV/1970, MZUSP não cat. (1); Monte Serrat, Salvador, 21/VII/1970, MZUSP não cat.(2). Espírito Santo - Vitória, XI/1964, MNRJ 10866(10); Praia de Camburi, idem, 24-25/I/1972, MZUSP não cat. (1). Rio de Janeiro - Atafona, VII/ 1963, MZUSP não cat. (176); Baía de Sepetiba, 28/ VII/1979, MCP 8275 (1); idem, 28/VII/1979, USU 00286 (9); idem, 28/VII/1974, USU 00298 (31); idem, 28/VII/1979, USU 00386 (6); Lagoa de Araruama, 20/X/1975, MNRJ 10444 (4);. São Paulo - São Paulo - Santos, VI/ 1914, MZUSP 819(9); Farol da Moela, Santos, VIII/ 1965, MZUSP não cat. (3); Ilha Bela, MZUSP 3649(188); Cananéia, 25-27/ VI/1964, MZUSP não cat. (1); Ubatuba, I/1976, MZUSP não cat. (4); Praia em frente à base Norte, Ubatuba, 19/V/1964, MZUSP não cat. (1); Praia da Ribeira, Ubatuba, 15/XII/ 1975, USU 00010 (4). Paraná Paranaguá, 25 40'S, 48 16'W, 01/II/1975, MZUSP não cat. (1). Santa Catarina - Florianópolis, II/1967, MCP 2446 (1); Bombinhas, 10/IV/1982, MCP 2983 (10); Garopaba, 20/I/1970, MCP 3557 (1); Tubarão, 
22-29/I/1971, MCP 5698 (1); Porto Belo, 03-04/XI/ 1973, MCP 7262 (1); Norte do Itajaí, 26 4’S, 48 08'W, 01/VI/1970, MZUSP não cat. (1); Itajaí, 26 53'S, $48^{\circ} 31^{\prime} \mathrm{W}, 16 / \mathrm{III} / 1970$, MZUSP não cat. (1);

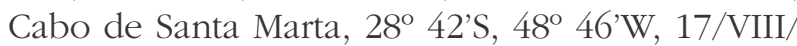
1970; MZUSP não cat. (1); Torres Araranguá, $29^{\circ}$ 13'S, $49^{\circ} 35^{\prime} \mathrm{W}, 30 / \mathrm{I} / 1972$, MZUSP não cat. (1). Rio Grande do Sul - Torres, 07-08/VI/ 1968, MCP 654(1); Torres-Tramandaí, 29 45'S, 49 $19^{\circ} \mathrm{W}, 16 / \mathrm{VIII} / 1968$, MZUSP não cat. (1); Lagoa de Tramandaí, 30/XI/ 1977, MZUSP 14272 (2); Ponte de Tramandaí, 06/ V/1974, MZUSP não cat. (1); Costa do Rio Grande do Sul, 14-30/VIII/1968, MZUSP não cat. (2). Uruguai - Montevidéu, Paralectótipo de Gerres aprion, MNHN 9457 (1).

\section{Eucinostomus melanopterus (Bleeker, 1863)}

Gerres melanopterus Bleeker, 1863: 44, pl. VIII, fig. 1 (Localidade típica: Guinea, Gold Coast, w. Africa, Ghana).
Eucinostomus poeyi Longley, 1935: 88 (Localidade típica: Cuba).

\section{Descrição e comentários}

Nadadeira anal com III +7 - 8 raios, sendo o terceiro espinho um pouco maior que o segundo. Presença de uma mancha negra na parte superior da nadadeira dorsal espinhosa com área brancoleitosa abaixo. Altura do corpo está contida 2,6 a 3,4 vezes no comprimento padrão. Ossos operculares lisos, com uma fina camada de escamas; 7 - 8 rastros na parte inferior do primeiro arco branquial. Nadadeira caudal apresenta 11 12 raios epaxiais acessórios, e 11 hipaxiais; 2 - 3 cecos pilóricos. Apresenta um padrão constante de coloração. A região dorsal mais escura do que a ventral. A nadadeira pélvica mais clara que as demais (Figura 4).

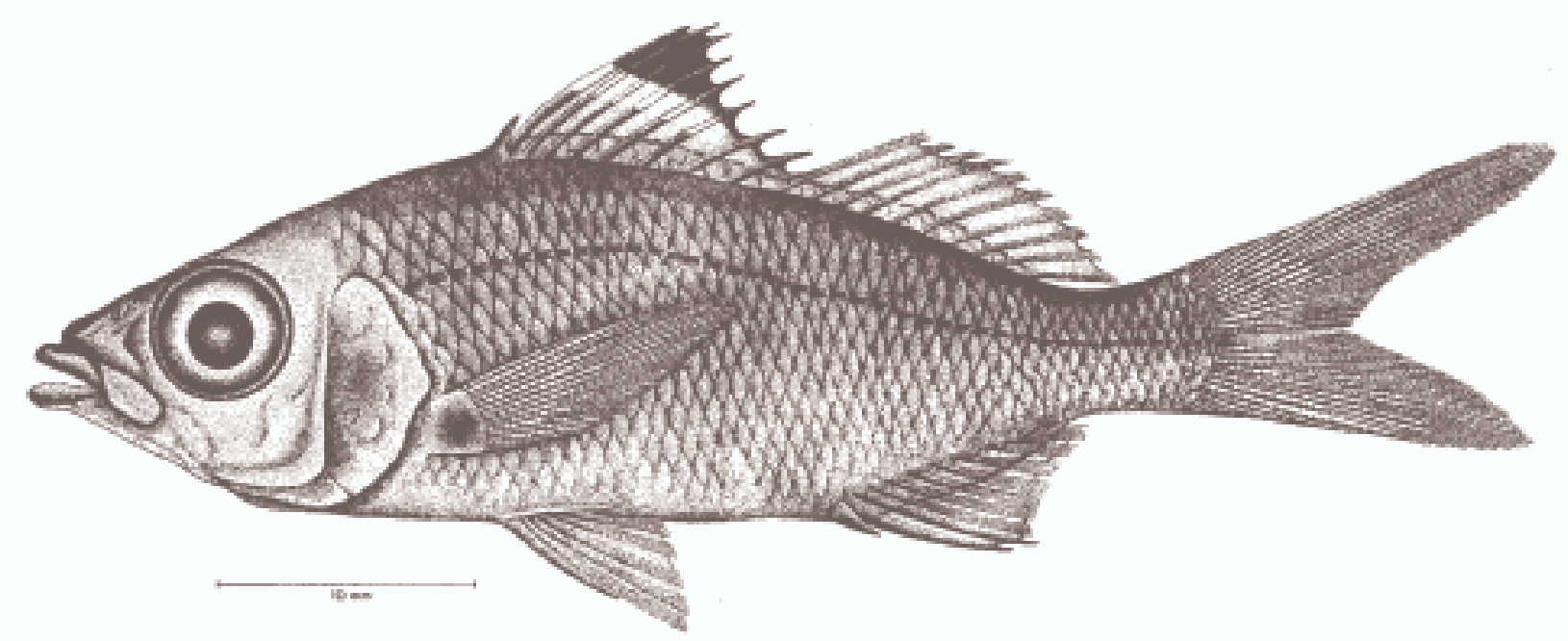

\section{FIGURA 4 - Vista lateral de Eucinostomus melanopterus}

Fonte: Bleeker, 1863.

A distribuição geográfica é ampla, ocorrendo no Atlântico norte e sul, no Golfo do México, mar das Antilhas e oeste da África. No Pacífico México e Costa Rica.

Os maiores espécimes capturados alcançam $150 \mathrm{~mm}$ de comprimento total. Segundo Menezes e Figueiredo (15), o corpo pode atingir $222 \mathrm{~mm}$. Os peixes jovens apresentam manchas e estrias escuras verticais nos lados do corpo, desaparecendo nos adultos. Vivem em fundo arenoso e vasoso, junto a manguezais. Denominada popularmente de carapicú, carapicú-açu, cacundo, escrivão e riscador.

O otólito sagitta tem a região anterior pouco recortada, enquanto a posterior é bem recortada, de forma elíptica, o ostium em forma de U, localizado bem acima próximo à região anterior (Figura 5). 


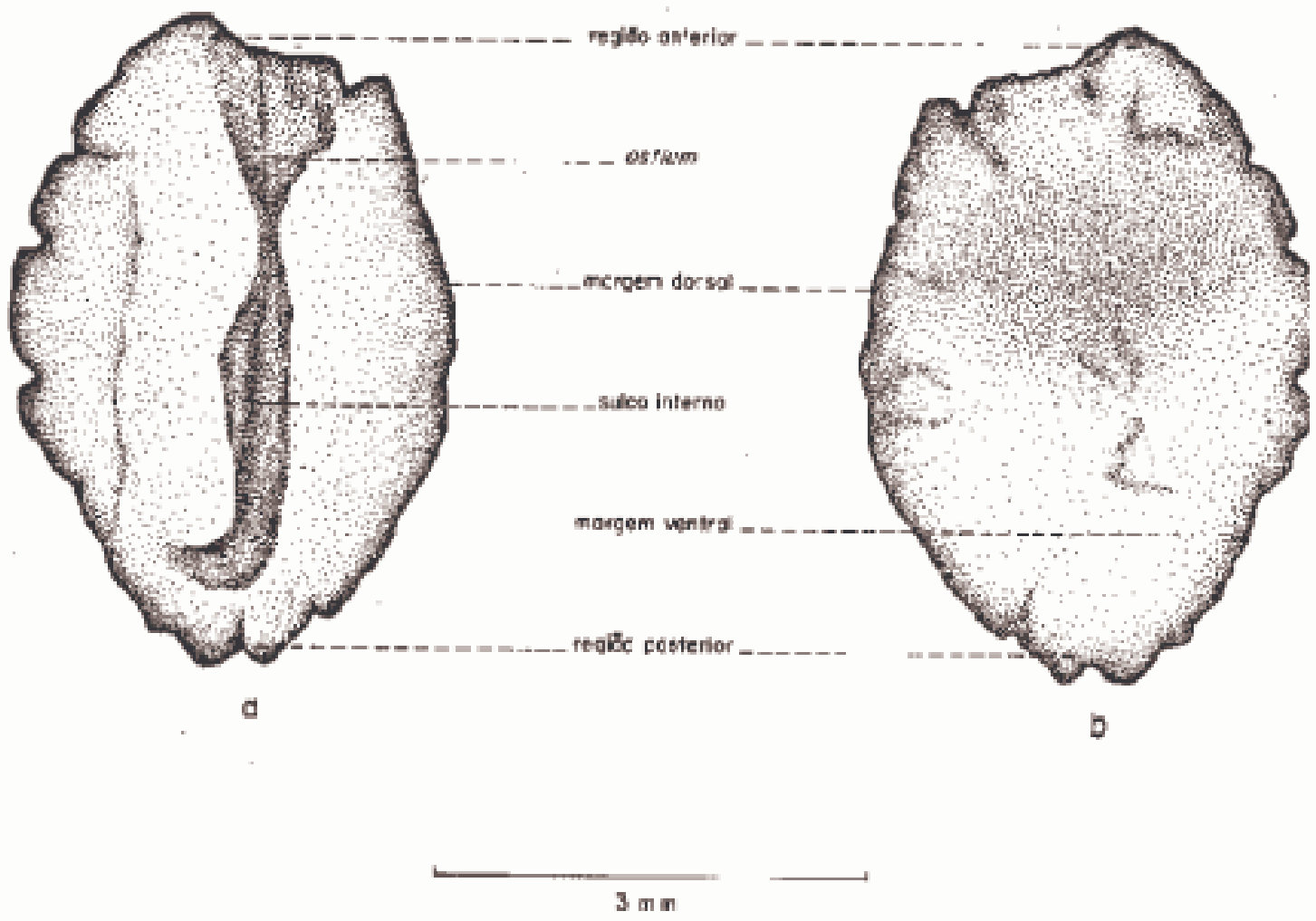

\section{FIGURA 5 - Vista lateral interna (a); vista lateral externa (b) do otólito sagitta de Eucinostomus melanopterus}

A análise do conteúdo estomacal revelou a presença de pequenos crustáceos (decápodebraquiúra), anelídeos (poliquetas). Segundo Longhurst (17), alimentam-se da fauna bêntica. Foram encontrados em salinidade de 16 a 36. Segundo Leon et al. (18), entre 1 a 4 e 29,4 a 36,4. Curran (19) comenta a captura desta espécie também em água doce (Rio Apuré e São Fernando do Apuré).

\section{Material examinado}

Cuba - Lectótipo de Eucinostomus poeyi MCZ 22014 (1); USNM 13060 (1). Haiti - 14/VII/ 1967, USNM 201686 (4). Porto Rico - Guajata River, MCZ 48617 (4). Honduras - 16/V/1975, FMNH 84996 (6). Panamá - Lat cove su of Buenaventura Cove, near Porto Belo, Colón, 18/IV/1971, GCRL 14025 (3): Darils Beach, Fort Sherman, $09^{\circ} 22^{\prime} 00^{\prime \prime} \mathrm{N}$, 7958'03"W, Colon, 04/XI/1973, GCRL 12154 (36);
$1 \mathrm{~km}$ E of Maria Chiquita at bridge crossing Porto Belo road Colán, 14/XI/1972, GCRL 10274 (2); Fort Sherman (Atlantic), MCZ 45526 (29); Bahia de Panamá, Pacífico, IX/1967, CCML não cat. (1). Trinidad - Blan Chisseusse, Mouth of Varra River, 23/IV/1967, AMNH 26393 (6). Colômbia - 20-21/ VIII/1969, UF 18986; Isla San Bernardo, ICN não cat. (1); Golfo Morrosquilo, ICN 334 (5); Brasil Calypso, 1961-1962, MNHN 399 (1). Rio Grande do Norte, Natal, XII/1912, AMNH 3856 (3). Pernambuco - Recife, II/1969, MCP 6984 (1); Ponte de Pedras, 04/XII/1974; MZUSP 74263 (2). Alagoas - Ponta Verde, Maceó, 12-14/XII/1973, MZUSP não cat. (2). Sergipe - Aracaju, VII/1961, MZUSP não cat. (6); Rio Sergipe, IV/1981, USU 00427 (12). Bahia - Praia Mocambo, Ilha Itaparica, 26/IV/1973, GCRJ 10999 (4); 01/XII/1974, MZUSP 742469 (6); 25/VIII/ 1972, MZUSP 1577 (42); 25/IV/1973, MZUSP não cat. (6); Tibras, Parque Interlagos, Salvador, GCRJ 10819 (1); Barra do Paraguassu-Mirim de Maragogipe, 10/XII/1974, MZUSP não cat. (1); Arembepe, 30/IV/1973, MZUSP não cat. (3); Rio 
de Janeiro - Atafona, VII/1963, MZUSP não cat. (19); Laguna de Marapendi, RJ, 23/III/1985, USU 00599 (1); Praia de Copacabana, 30/XI/1983, USU 00426 (1). São Paulo - Ubatuba, VII/1971, MZUSP 10299 (5); Santos VI, MZUSP 869 (1); MZUSP não cat. (1); São Sebastião, 1915, MZUSP 2410 (2); Cananéia, VI/1971, MZUSP não cat. (1); Ilha Bela, 06/III/1962, MZUSP não cat. (1); Iguape, 1902, MZUSP 2408 (1). Santa Catarina - Palhoça, MCP 5227 (1); Porto Belo, 03-04/XI/1973 7207 (1); Sambaqui, Florianópolis 14/VII/1977, MZUSP 13329 (1). Rio Grande do Sul-Torres, 07-08/VI/1968, MCP 659 (1).

\section{Eucinostomus gula (Quoy \& Gaimard, 1824)}

Gerres gula Quoy \& Gaimard, 1824: 293 (Localidade típica: Martinica).

Eucinostomus gula, Goode, 1876: 39 (Localidade típica: Brasil).

\section{Descrição e comentários}

Sulco pré-maxilar interrompido por uma faixa de escamas. A altura do corpo está contida 2,3 - 2,6 vezes no comprimento padrão. Cabeça está contida 2,8 e 3,1 no comprimento padrão. Ossos operculares lisos e cobertos com fina camada de escamas. Nadadeira pélvica não ultrapassa o ânus; 7 - 8 rastros na parte inferior do primeiro arco branquial; 37 - 45 escamas na linha lateral; 8 - 10 abaixo. Nadadeira dorsal IX +10 ; nadadeira anal III $+7 ; 10$ raios epaxiais e 10 hipaxiais acessórios. A coloração é prateada uniforme com tonalidade pardacenta. Pigmentos escuros na extremidade dos espinhos da nadadeira caudal e as demais nadadeiras claras (Figura 6). Os maiores espécimes examinados mediram $170 \mathrm{~mm}$ de comprimento total. Denominados popularmente de carapicú, carapau, carapicu-açu.

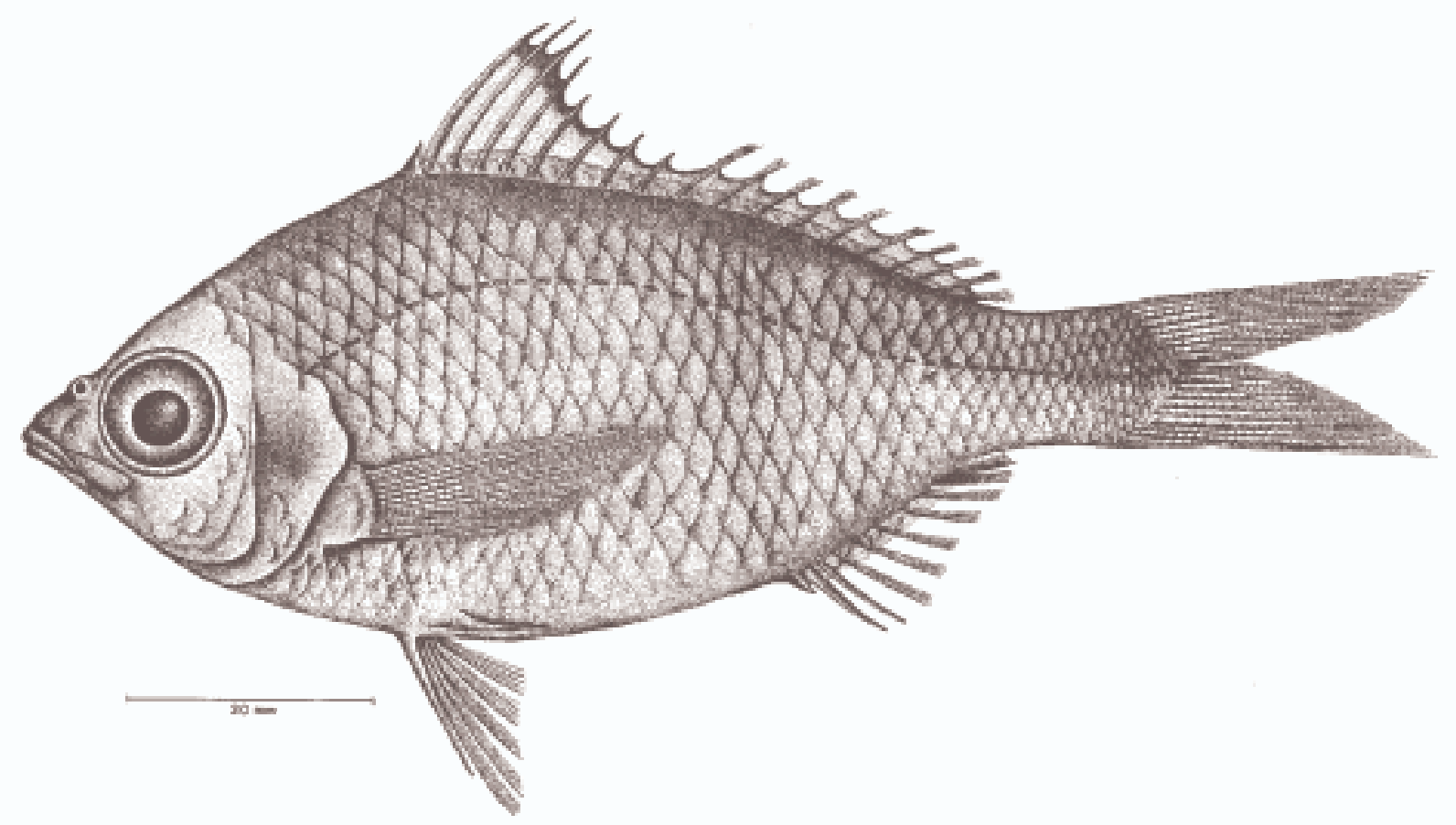

FIGURA 6 - Vista lateral de Eucinostomus gula

Fonte: Quoy \& Gaimard, 1824.

Distribui-se no Atlântico desde New Jersey até o Rio Grande do Sul, inclusive nas Bermudas, Golfo do México e Mar das Antilhas, no Pacífico, Equador. Segundo Cervigon (20), Menezes e Figueiredo (14) e Leon et al. (18), chega até a Argentina, e Leon et al. (18), ocorre também no México.

O otólito sagitta tem forma elíptica, região anterior expandida, margem pouco recortada, ostium relativamente pequeno, menor 
que nas demais espécies do gênero, e mais profundo do que em E. aprion (10) (Figura 7a, b). Alimentam-se de pequenos moluscos, crustáceos (copépodes, anfípodes, decápodes, isópodes), anelídeos (poliquetas), algas e fragmentos de matéria orgânica. Ocorre em salinidade de 15,56 a 36 onde vive próximo ao fundo arenoso e vasoso.

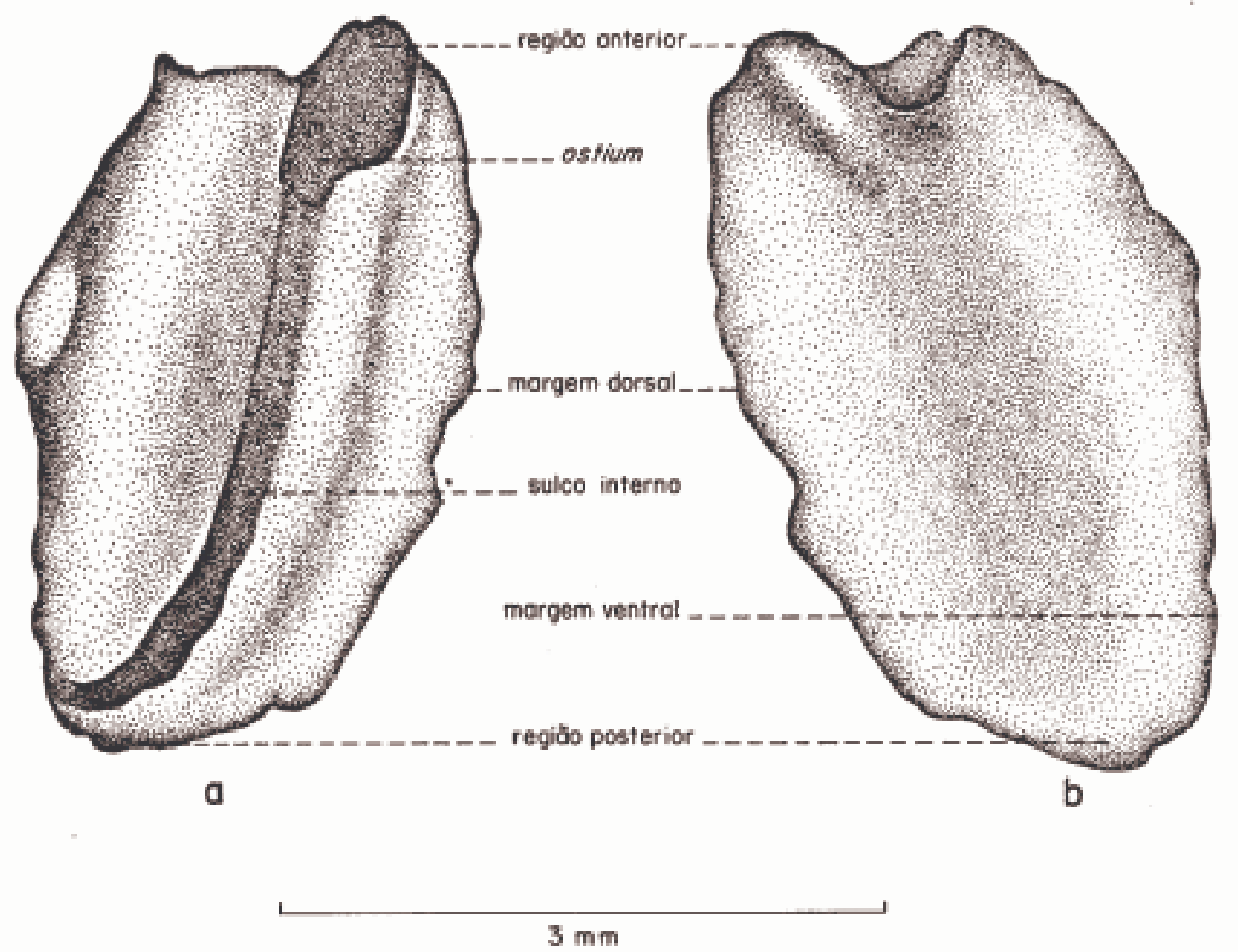

\section{FIGURA 7 - Vista lateral interna (a); vista lateral externa (b) do otólito sagitta de Eucinostomus gula}

\section{Material examinado}

Bermudas - VIII/1937, MCZ 32921 (5); 1980, ZMK 549 (1); RMNH 1096 (1); St. Thomas, ZMK 548 (1). Estados Unidos - Syntype de Diapterus homonymus, Clear Water Habor, Flórida; USNM 23639 (3); North Carolina, Hussakoff, AJ 17764 (3); South Carolina, ChalE Country, Long Point towing North in Wando River, 30/X11 AMNH Uncat (5). Golfo do México - Atlantic Nort Back Bay Creek west side of Huy 18: before Village of Bokeelia, Pine Island, Lee Country, [26 $~ 82^{\circ} 09^{\prime} \mathrm{W}$ ], 31/VIII/1977, NMC 78-0360 (49); Taylor Co., Ac Beach, 24/IXI1966, UF/FSU 15106 (10). Cuba Havana, 13/VIII/1946, USNM 132978 (1); 1912, AMNH 336: 1912, AMNH 1592 (1); SU 63931 (3);
Santiago de Cuba, IV/1 AMNH 17264 (1). Haiti1927, USNM 178752 (1); índias Ocidentais -USNM 41289 (1). Porto Rico - San Juan Santurce, 21/VII/ 1914, AMNH 8881 (2); San Antonio Bridge, 11/ VII/1' AMNH 4896 (10); Paio Seco Pt. VII/1914, AMNH 5568. Panamá- 27/I/1924, AMNH 11420 (15); 04/III/1937, USNM 144461 Ambrosia Bight (Atlantic), MCZ 43488 (6); Atlântico, IV/1975, CI não cat. (1). Jamaica - Small strem in Negil, MCZ 58330 (1). British Guianas - 01/IXI1961, BMNH 67-64 (4); Oregon Sta $22^{\circ} 35^{\prime} 08^{\circ} 15^{\prime} \mathrm{N}, 58^{\circ} 28^{\prime} \mathrm{W}, 29 /$ VIII/1958, FMNH 67599 (3). Martinica - Lectótipo de Gerres gula, MNHN 9461 (1); paralectótipo Gerres gula MNHN B2997 (1). Barbados - 08/II/ 1968, NMC 68244 (1). Windward - 08/VII/1932; USNM $178633(1),\left[14^{\circ} \mathrm{N} 62^{\circ} \mathrm{W}\right.$ ]. Costa Rica -Limon, 
19/11/1908, USNM 94163 (1). Trinidad - VIII/1937, MCZ 34678(2). Equador - Punto Carnero, prox. Guayaquil, 05/IX11962, N 68-1933 (97). Venezuela - N. Esparta, Isla Margarita, Boca Dei Rio, 20/II/ 1977, GCRL 1, 15802 (1); Isla Larga, Punta Este dei Archipiélago Los Roques, Dependência Federal, 05/ VIII/1963, USU 00409 (3). Colômbia - Isla de San Bernardo, 20/VI/1961, ICN 537 (4). Brasil Paralectótipo de Gerres gula, MNHN 9463 (3); MNHN, 1975280 (2); MNHN 5761 (2); MNHN 1975399 (1); MNHN H 1975-1185 (1); MNHN 488 (1); MNHN 489 (1). Ceará - Fortaleza, Rio Coco, III/ 1945, MNRJ 10868 (5); Morro Branco, J, próximo de Beberibé, CE, MZUSP 720769 (2); MZUSP 2401 (2); Sergipe - Rio Sergipe, local 2, Grea 2, X/1981, USU 00414 (3). Bahia - MNHN 985 (1); MNHN 508 (1); MNHN 151 (1); MNHN 2805 (1); MNHN 2235 (1); Abrolhos, XII/1978, MNRJ 10702 (77). Espírito Santo - Ilha da Conceição, próxima a Vitória, XI/1964, MNRJ 10886 (10); Praia de Camburiu, Vitória, 24-25/I/1972, MZUSP não cat, (1). Rio de Janeiro - MNHN 205 (1); MNHN 206 (1); MZUSP 3157 (1); Laguna da Tijuca, 12/V/1980, MNRJ 10790 (4); Baía da Guanabara, 04/ 1X/1982, MNRJ 10860 (34); Lagoa de Saquarema, VII/1980, MNRJ 10780 (2); Ilha Grande, MNHN $1975-$ 280 (2). São Paulo - Ilha da Moela, Santos, 18/IV/ 1962, MZUSP não cat. (1); 245'ㅇ's e $47^{\circ} 00^{\prime} \mathrm{W}$, Enseada de Bertioga com Itaguaré, 30/V/1970. MZUSP não cat. (2); Ubatuba, 24/V/1964, MZUSP não cat. (1). Santa Catarina - Florianópolis, II/1967, MCP 2394 (1); II/1967, MCP 2422 (1); II/1967, MCP 2439 (1); X/1968, MCP 3149 (1); Garopaba, 18/I/ 1970, MCP 3543 (1); Praia de Perequê, 04/VIII/1978, MCP8250 (1); 26언 e 4802'W, Paranaguá, Paraná,
PR, 15/VII/1970, MZUSP não cat. (1); 2540'5 e 48¹6'W; idem, 01/II/1975, MZUSP não cat. (3).

\section{Eucinostomus lefroyi (Goode, 1874) Combinação nova}

Diapterus lefroyi Goode, 1874: 123 (Localidade típica: Bermudas).

Eucinostomus productus Poey, 1875: 129 (Localidade típica: Havana).

\section{Descrição e comentários}

Nadadeira anal II + 7 - 8 raios; mancha escura sobre a pupila. Corpo baixo, altura do corpo contida 2,3 a 4,0 vezes no comprimento padrão; distância prédorsal sempre maior que a altura do corpo, sulco prémaxilar contínuo; olhos relativamente grandes com uma mancha escura sobre a pupila, principalmente nos jovens ou recém-coletados. Ossos operculares finos, cobertos de escamas com bordas lisas; 7 - 8 rastros finos e pequenos na parte inferior do primeiro arco branquial; nadadeira anal +7 - 8 raios; 2 cecos pilóricos. Possuem um padrão de coloração definido, prateado, com dorso mais escuro que a região ventral, quando jovens ou recém-coletados com manchas e barras diagonais escuras na parte superior do corpo. Nadadeira dorsal com pigmentação escura na parte superior e espinhos; nadadeira anal com pigmentação escura em toda a sua extensão. Os maiores exemplares examinados medem $173 \mathrm{~mm}$ de comprimento total. Vulgarmente conhecidos como carapicú-açu, carapau e carapicú-branco (Figura 8).

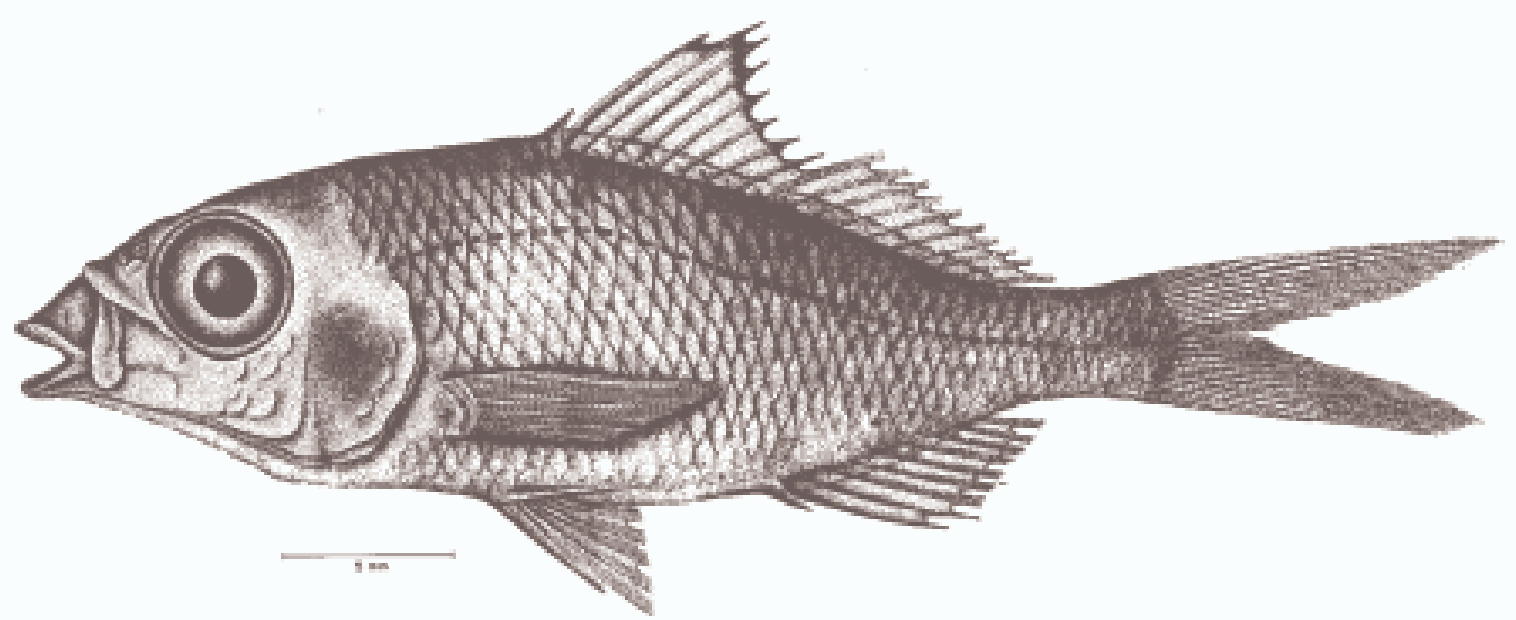

FIGURA 8 - Vista lateral de Eucinostomus lefroy

Fonte: Goode, 1874. 
Apresenta ampla distribuição geográfica, ocorrendo no oceano Atlântico desde New Jersey ao Sul do Brasil, inclusive nas Bermudas e Antilhas.
O otólito sagitta tem a forma elíptica, região anterior afilada com reentrâncias, ostium estreito, grande e raso, semelhante ao de E. aprion (10) (Figura 9a, b).

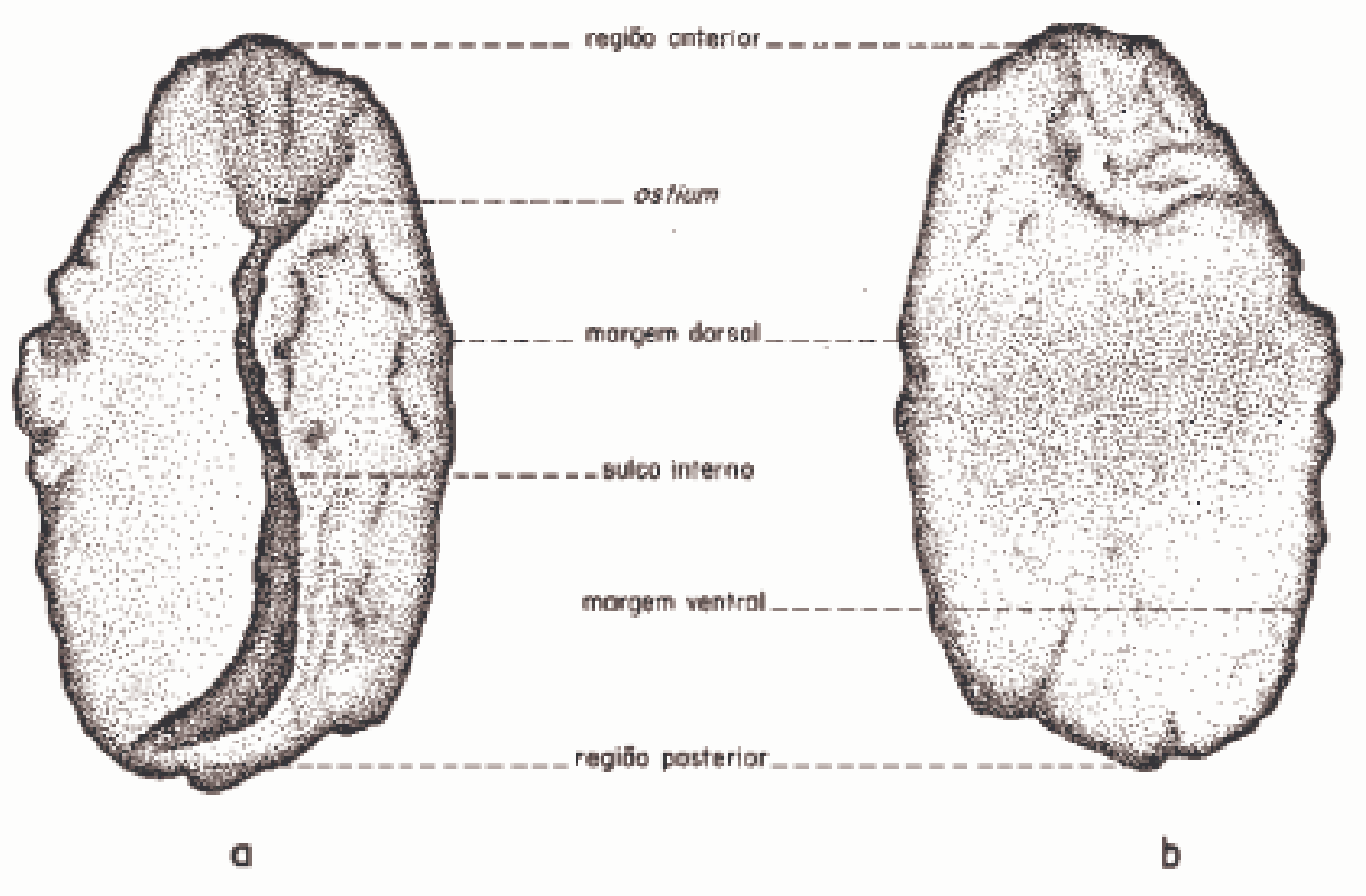

$3 \mathrm{~mm}$

\section{FIGURA 9 - Vista lateral interna (a); vista lateral externa (b) do otólito sagitta de Eucinostomus Lefroyi}

A análise do conteúdo estomacal revelou a presença de pequenos moluscos, crustáceos (copépodes) e anelídeos (poliquetas). Quanto à salinidade, foram encontrados espécimes entre 16 a $36 \mathrm{em}$ água doce. Vive em fundo arenoso e vasoso, ocorrendo simpatricamente com Eucinostomus aprion, sendo bastante similares morfologicamente.

\section{Material examinado}

Bermudas - Síntipo de Diapterus lefroyi, USNM 16760 (2). Estados Unidos - New Jersey,
Brigantine National Wildlife Refuge, Reed Bay, AMNH 56057 (5); Virginia, Rudee Inlet, Virginia Beach, 123/VI/1976' AMNH uncat (2); Mobile Co., Dauphin Island, Alabama, 11/X/1975, AMNH 35729 (1); Florida, Breward/Indian R. cos Sebastian Inlet, Lat. $27^{\circ} 51^{\prime} \mathrm{N}$, Long. $80^{\circ} 27^{\prime} \mathrm{W}, 14 / \mathrm{VI} / 1976$, TCWC 2893.5610). Cuba - Holótipo Eucinostomus productus USNM 13038. St. Eustatius - RMNH 9453 (2) (Mar do Caribe). Guatemala - Approy $15^{\circ} 44^{\prime} 45^{\prime \prime} \mathrm{N}, 88^{\circ} 37^{\prime} 45^{\prime \prime} \mathrm{W}$, Bahia de Matias de Galvez, Izabal 28/V/1969, GCRL 4407 (5). Panamá Miraflores spillway dam and bridge, 02/IV/1973, I 11595 (1); $1 \mathrm{~km}$ E of Maria Chiquita at bridge crossing Porto road, Colán, 14/XI/1972, GCRl 21136 
(4); Taipon Club Boat B Atlantic coast of Panamá (fresch water), MCZ 59779 (1). Venezuela - Isla Cubaqua pta. Charaquto to pta. Brasil, nueva Esp Isla Cubagum, 16/II/1977, GCRl 21127 (4). Colômbia - Isla de San Bernardo, ICN não cat. (1). Brasil - $1^{\circ} 30^{\prime} 5^{\prime \prime}, 1^{\circ} 33^{\prime} \mathrm{W}$ a $43^{\circ} 40^{\prime} 5 \mathrm{~W}, 43^{\circ} 46^{\prime} \mathrm{W}, 07 /$ IV/1957, near Parni City, MCZ 46B56 (2). Pernambuco - Itamaracá, Mar de Dentro, 11/V/ 1971, MZ 13596-614 (19); Ponta de Pedras, 04/II/ 1969, MZUSP não cat. idem, 30/VIII/1970. MZUSP não cat. (2). Alagoas - Ponta verde, Maceió, 12 e 14/XII/1973, MUZ5P não cat. Bahia - Arembepe, 23/VII/1972, MZUSP 1570 (7); Parque Interlagos, 27 /VIII/1972; MZUSP 1580 Praia de Arembepe, 26/VIII/1972, MZUSP 1579 (5); 1245'00'5, 38¹0'00'W, 26/VIII/1982, GCR19404 (3), de Itaparica, Rio Penha, 25/IV/1973, MZUSP não cat. (13). Rio de Janeiro - Paia de Copacabana, posto 6, 07/X11983, L 00431 (1). São Paulo - Ilha Bela, 06/II/1962, MZUSP não cat. (1). Rio Grande do 5ul - Ponta dos Pescadores, 18/II/1981, USU 00323 (2).

\section{Discussão}

A análise dos espécimes-tipo e de um grande número de exemplares de Gerreidae procedentes de diversas regiões geográficas permitiu avaliar as espécies brasileiras de Eucinostomus, considerando-se como válidas Eucinostomus aprion, Eucinostomus melanopterus, Eucinostomus gula e Eucinostomus lefroyi, anteriormente tratadas por Andreata $(8,9,10)$ como pertencentes ao gênero Gerres Quoy \& Gaimard, 1824 .

A espécie Gerres aprion descrita por Cuvier, 1829: 188 apresenta o corpo idêntico ao de Eucinostomus argenteus Baird \& Girard in Baird, 1855: 345 , sendo por isso considerada como um sinônimo.

O exame do sintipo de Diapterus lefroyi e de um grande número de exemplares de várias coleções evidenciou um conjunto de características, tais como que a nadadeira anal com II $+7-8$ raios, mancha escura sobre a pupila, altura do corpo; o que distância pré-dorsal, o que diagnosticam o gênero Eucinostomus, sendo, portanto, proposta a transferência de Ulaema lefroyi para Eucinostomus.

Os exemplares tipos procedentes da Martinica, Windward e do Brasil de Eucinostomus gula apresentam as mesmas características. Notouse que os espécimes do oceano Pacífico (Galápagos) o diâmetro dos olhos é um pouco maior e a altura do corpo um pouco mais baixo do que naqueles procedentes do oceano Atlântico.

A análise realizada das proporções morfométricas revelou índices altamente significativos para as espécies estudadas de Eucinostomus (8, 10). Matheson e McEachran (6) referem-se a $E$. argenteus como um complexo, em virtude das dificuldades em diferenciá-lo das demais espécies do gênero Eucinostomus.

No exame geral dos otólitos sagitta a forma é elíptica para todas as espécies. Os caracteres morfológicos apresentam pequenas diferenças entre as espécies observadas. O ostium na maioria das espécies de Eucinostomus é pequeno e raso, com exceção de E. lefroyi no qual é maior. O sulco interno variou sendo em E.aprion largo e nas demais espécies estreito.

A distribuição geográfica das espécies estudadas é ampla, ocorrendo ao longo da costa das Américas, estendendo-se de norte a sul, sendo abundantes na América Central. No Brasil ocorre desde o Nordeste até o Sul, tendo ampla distribuição e vivendo em diferentes biótopos, em variáveis ambientais diferentes, o que provavelmente se reflete nas características morfoanatômicas dessas espécies (9).

\section{Agradecimentos}

Agradeço à Professora Doutora Jeanete Maron Ramos Chanceler e Diretora de Pesquisa e ao Professor Doutor Carlos Potsch ex-Reitor da Universidade Santa Úrsula pelo apoio e incentivo às nossas pesquisas. A todos os curadores pelo empréstimo ou por ter permitido a consulta das coleções examinadas.

\section{Referências}

1. Böhlke JE, Chaplin CCG. Fishes of the Bahamas and adjacent tropical waters. Acad Nat Sci Philad; 1968.

2. Böhlke, JE, Chaplin, CCG. Fishes of the Bahamas and adjacent tropical waters. Acad Nat Sci Philad; 1993. 
Sobre as espécies de Eucinostomus Baird \& Girard in Baird, 1855 that they occur in brazilian waters (Actinopterygii, Perciformes, Gerreidae)

3. Cyrus DP, Blaber SJM. A biologia reproductiva de Gerres (Teleostei) Bleeker 1859, em estuários Natal. J Biol dos peixes. 1984; 24:491-504.

4. Eschmeyer WN. Catalog of fishes. Special Publication. San Francisco: California Academy of Sciences; 1998. $3 \mathrm{v}$.

5. Nelson JS. Fishes of the world. $3^{\text {rd }}$ ed. New York: John Wiley and sons; 1994.

6. Matheson JR, Mceachran JD. Taxonomic studies of the Eucinostomus argenteus complex (Pisces: Gerreidae): Preliminary studies of external morphology. Copeia. 1984; 4:893-902.

7. Froese R, Pauly D, editor. Fishbase 2006. [serial on the Internet]. [cited 2006 Jan. 15]. Available from URL: http://www.fishbase.org.

8. Andreata JV. Revisão das espécies de Gerreidae (Perciformes, Percoidei) que ocorrem no litoral brasileiro e relações entre os gêneros. [tese Zoologia]: Universidade de São Paulo. São Paulo; 1987.

9. Andreata JV. Estudo taxonômico das espécies de Gerres Quoy \& Gaimard, 1824 (Pisces, Perciformes, Gerreidae) que ocorrem em águas brasileiras. Acta Biologica Leopoldensia. 1989a; 11(1):87-128.

10. Andreata JV. Sobre a osteologia das espécies de Gerres Quoy \& Gaimard, 1824 (Pisces, Perciformes, Gerreidae) que ocorrem em águas brasileiras. Acta Biologica Leopoldensia. 1989b; 11(2):165-202.

11. Taylor WR. A enzyme method of clering and staining small vertebrates. Proc U S natn Mus. 1967; 122(3569):1-179.
12. Nolf JT. Contribuition a l'étude des otolithes des poissons (1) morphologie comprarée des otolithes (Sagittae) des Dentex de la Mediterranée et de l'Atlantique tropical africain. Bull Inst Sci nat Belg. 1979; 51(9):1-14.

13. Chao LN. A basis for classifying western Atlantic Sciaenidae (Teleostei, Perciformes). NOAA Tech Rep- NMFS Tech. Circular. 1978; 414:1-64.

14. Zavala-Camin LA. Introdução aos estudos sobre alimentação natural em peixes. Maringá: Nupélia, EDUEM; 1996.

15. Menezes NA, Figueiredo JL, Teleostei. In: Manual de peixes marinhos do sudeste do Brasil. São Paulo: Mus. Zool, USP/CNPq. 1980; 4(3).

16. Springer VG, Woodburn, KD. An ecological study of fishes of Tampa Bay area. Fia St Cons Prof Pap. 1960; 1:1-104.

17. Longhurst, A. R. The food of the demersal fish of west Africa estuary. J Anim Ecol. 1957; 26(2):369-387.

18. Leon AA, Yánez-Arancibia A, Linares FA. Taxonomia diversidad, distribuicion y abundancia de los moiarras de La Laguna de Terminos, Campeche (Pisces: Gerreidae). An Inst Cienc del Mar y Limnol. 1982; 9(1):213250.

19. Curran AWA. Systematic revision of the gerrid fishes refered to the genus Eucinostomus, with a discussion of their distribution and speciation. Michigan: Univ Michigan; 1942.

20. Cervigon F. Los peces marinos de Venezuela. Caracas: Fund La Salle Cienc Nat. 1966. 2:449949. 